\title{
Monte Carlo optimization simulation of neutron shielding performance of iron/polyethylene combined structure
}

\author{
Yinghong Zuo*, Jinhui Zhu, Shengli Niu, Honggang Xie, and Peng Shang \\ Northwest Institute of Nuclear Technology, Xi'an, 710024, China
}

\begin{abstract}
This study aims to get the optimization neutron shielding design of iron/polyethylene combined shield structure. The neutron transmission coefficient with various energies for different thickness of iron and polyethylene combined shield structure were calculated by using Monte Carlo method. The simulation results show that the optimization effect of iron/polyethylene combined shield is not obvious when the neutron energy is low or the shield is thin, there is an optimal thickness ratio of iron to polyethylene adopted to get the best neutron shielding performance when the energy of neutron source is above $2 \mathrm{MeV}$ and the total thickness of combined shielding structure is more than $20 \mathrm{~cm}$. The optimal thickness ratio of iron to polyethylene increases with the increasing energy of neutron source; with the increasing of neutron source energy ranging from $4 \mathrm{MeV}$ to $14 \mathrm{MeV}$, the optimal thickness ratio of iron to polyethylene trends from 0.11 to nearly 1.6 .
\end{abstract}

\section{Introduction}

With the deepening research of nuclear technology and its application, the radiation protection is becoming more and more important. Neutron shielding is an important issue in the field of radiation protection[1-2]. In the process of shielding design, it is necessary to pay attention to whether the shielding performance of material meets the requirements of radiation protection, but also necessary to consider the factors such as the thickness, size and volume of shielding materials[3-4]. The numerical simulation or experimental researches of neutron shielding for various materials has been carried out at home and abroad. Zeng Xinmiao calculated the transmission coefficients of typical materials with different thickness for neutron with different energy[5]; Yang Wenfeng used the method of splitting cross section, according to the empirical formula, calculated the ${ }^{241} \mathrm{Am}-\mathrm{Be}$ fission spectrum neutron shielding performance of 4 kinds of composite materials[6]. Han Zhongwu studied the neutron shielding performance of tungsten nickel alloy[7]. Shen Zhiqiang studied the shielding performance of cement based materials and polyethylene (PE) multi-layer composite shielding structure for $14.8 \mathrm{MeV}$ neutrons. The research results show that the composite shielding structure has a stronger capacity than the single structure[8]. Yang Huazhong et al, by using Monte Carlo simulation method, calculated the

* Corresponding author: zuoyinghong@nint.ac.cn 
$\mathrm{T}(\mathrm{D}, \mathrm{n})^{4} \mathrm{He}$ neutron transmission coefficient of natural iron spherical shell with different thicknesses, which can provide reference for selection of the first fast neutron source shielding material[9]. Luo Peng et al had carried out the simulation and comparison researches of $\mathrm{T}(\mathrm{D}, \mathrm{n})^{4} \mathrm{He}$ neutron shielding performance of three kinds of shielding structure: iron- PE- boron carbide, copper- PE- boron carbide and tungsten- PE- boron carbide, wherein the metal and PE layer thickness values are selected for a single value[10].

As the most typical shielding materials, iron and polyethylene are often used together. At present, the determination of the combination thickness of the two is mainly dependent on experience. There is no theoretical research work on the optimization design of the two combination thickness. How to optimize the neutron shielding effectiveness of shielding material at the condition that shielding materials have certain constrains, is one of the key points in the design of neutron shielding. In this paper, the Monte Carlo method is used to simulate the neutron shielding performance of iron-polyethylene composite materials. The transmission coefficient of neutrons with different energy of iron and polyethylene composite materials with different thickness is calculated out to obtain the optimal size combination scheme, which can provides a basis reference for neutron shielding design.

\section{Physical model and method}

The physical model of iron-polyethylene combined shield is established as shown in figure 1. Single energy narrow beam neutron source is incident perpendicularly toward the shield, the distance between neutron source and shield is $10 \mathrm{~cm}$, and the neutron source and shield is surrounded by vacuum.

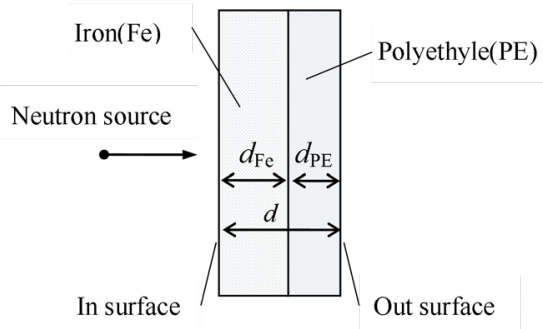

Fig. 1. Scheme of the physical model.

The shape of the shield is a circular plate with a radius of $1.5 \mathrm{~m}$. The total thickness of the combined shield is $d$, where the thickness of the iron layer is $d_{\mathrm{Fe}}$, and the proportion of the thickness of the iron layer in the total thickness is set from 0 to $100 \%$. When the proportions are equal to 0 or $100 \%$, respectively, the combined shield becomes a single polyethylene or iron alone. In the practice of radiation shielding, a lead layer is usually added after the polyethylene to absorb the secondary gamma rays generated by the interaction between the neutron and iron or polyethylene. Taking into account that the absorption of the secondary gamma rays is relatively easy, the simulation in the paper does not involve the shielding calculation of the secondary gamma rays absorbed by lead.

The shielding properties of materials are usually characterized by the transmission coefficient of neutron, the smaller the transmission coefficient is, the better the shielding performance is. The transmission coefficient $I_{\mathrm{t}}$ is defined as the ratio of the number of neutrons transmitted by the exit surface $N_{\mathrm{t}}$ to the number of neutrons incident by the incident surface $N_{0}$ :

$$
I_{\mathrm{t}}=N_{\mathrm{t}} / N_{0}
$$

The MCNP 4B program is used to calculate the transport of particles[11]. The program generates pseudo random numbers, and then the parameters such as target nucleus, collision 
type and collision exit angle are sampled from pseudo random numbers to track the motion process of a large number of particles in the shielding body, the number of neutrons that can penetrate the shielding surface $N_{\mathrm{t}}$ can be counted finally by using tally F1 card. The number of the simulated incident neutrons is $N_{0}=1 \times 10^{7}$.

In addition to the study of the shielding performance of the single neutron, the fission neutron shielding performance of the composite material is also simulated. The fission neutron spectrum is described by Maxwell distribution spectrum, and the expression of the energy spectrum distribution is distribution is

$$
\mathrm{d} N / \mathrm{d} E=C E^{1 / 2} \mathrm{e}^{-E / a}
$$

where, $C$ is the total number of fission neutrons; $\mathrm{d} N / \mathrm{d} E$ is the number of neutrons within the unit energy interval, which is a function of the energy of the fission neutron; the constant $a$ is related to the type of nuclear material nuclides and the energy of the neutron that induce fission, the nuclear material is selected to be ${ }^{239} \mathrm{Pu}$ and the energy of neutron that induce fission is $1 \mathrm{MeV}$ in this paper, that is $a=1.3$.

\section{Simulation result and discussion}

According to the above physical model, the total thickness of the combined shield is taken as $20 \mathrm{~cm}, 40 \mathrm{~cm}, 60 \mathrm{~cm}$ and $80 \mathrm{~cm}$ in the simulation, and the transmission coefficients of different energy neutrons are obtained under different combinations. The staistical error of the simulation results is not more than $5 \%$.

\subsection{The optimum thickness ratio at different total thickness}

Figure 2 shows that the transmission coefficients of neutron with different energy change with the proportion of the thickness of the iron layer in the combined shield for four different total thicknesses. The $p_{\mathrm{Fe}}$ is the proportion of the iron thickness to the total thickness of combined shield. As can be seen from figure 2, when the total thickness of the combined shield is the same, the ratio of the thickness of the iron to the polyethylene is different, and the transmission coefficient of the neutron is different. It can be seen from figure 2 (a), there is no optimal thickness ratio when the neutron energy is low. For example, when the neutron energy is $0.1 \mathrm{MeV}$, the shielding performance of the combination of iron and polyethylene is worse than that of a single polyethylene material. This is because the inelastic scattering of neutron and iron can happen only when the neutron energy is greater than $0.85 \mathrm{MeV}$. When the neutron energy is low, the interaction of the inelastic scattering between neutron and the iron shield is not sufficient, so the slowing down effect of iron on the neutrons is not ideal[11].

From figure $2(\mathrm{~b}) \sim(\mathrm{d})$, when the neutron energy is high, with the increase of the proportion of iron layer thickness to total thickness of combined shield, the neutron transmission coefficient of the combined shield decreases firstly and then increases gradually. That is, the shielding performance of the combined shield is gradually improved and then weakened, and there will be a best thickness ratio of iron to polyethylene that makes the best shielding performance of the combined shield. Taking the total thickness of combined shield is $d=60 \mathrm{~cm}$ as shown in figure 2(d) as an example, when using polyethylene or iron as a single shield with thickness of $60 \mathrm{~cm}$, the transmission coefficient of $14 \mathrm{MeV}$ neutrons are $2.30 \times 10^{-2}$ and 0.217 ,respectively; When the thickness ratio of iron to polyethylene in the combined shield is $0.62: 0.38$, the transmission coefficient of 14 $\mathrm{MeV}$ neutrons drops to $2.54 \times 10^{-3}$, i.e., with the case of $14 \mathrm{MeV}$ neutrons and total thickness of $60 \mathrm{~cm}$, the optimum thickness ratio of iron and polyethylene is about 0.62:0.38=1.6:1 which can make the combined shield show best shielding performance. 
From figure $2(\mathrm{~b}) \sim(\mathrm{d})$, it can be concluded that for the fission neutron with an average energy of $2.1 \mathrm{MeV}$, mono-energetic neutron with energy of $5 \mathrm{MeV}$ and with energy of 14 $\mathrm{MeV}$, the optimum thickness ratio of iron and polyethylene is about 0.3:0.7 $(=0.43: 1)$, 0.4:0.6 $(=0.67: 1)$ and $0.62: 0.38(=1.63: 1)$ respectively when the neutron shielding performance of combined shield is optimal. When the neutron energy is equal to or greater than $1 \mathrm{MeV}$, the optimum thickness ratio of iron to polyethylene in the combined shield increases with the increasing of neutron energy.
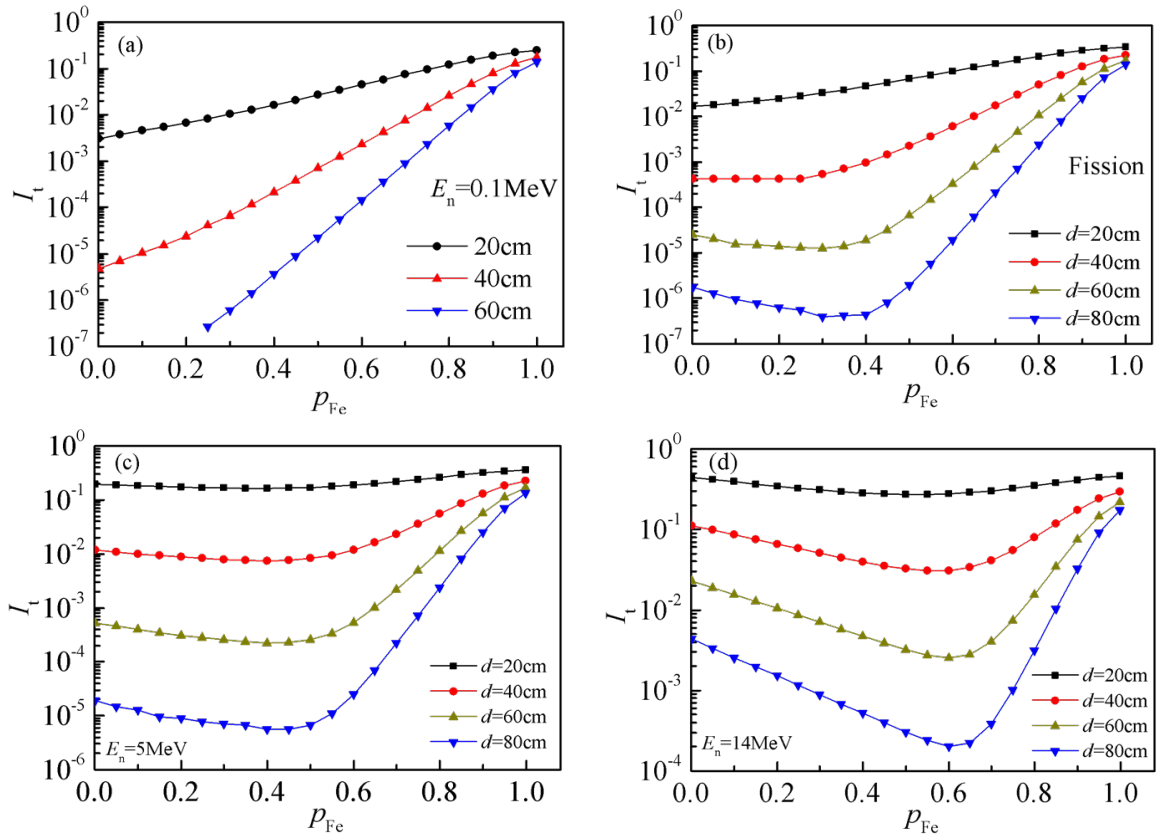

Fig. 2. Neutron transmission coefficient versus iron thickness proportion in combined shield structure. (a) $E_{\mathrm{n}}=0.1 \mathrm{MeV}$, (b) Fission neutron, (c) $E_{\mathrm{n}}=5 \mathrm{MeV}$, (d) $E_{\mathrm{n}}=14 \mathrm{MeV}$.

The neutron transmission coefficient of combined shield is affected by macroscopic parameters such as the neutron energy, the total thickness of the combined shield and the thickness ratio of the composite material, and is microscopically dominated by the interaction cross section between neutrons and radionuclide such as ${ }^{1} \mathrm{H},{ }^{12} \mathrm{C}$ and ${ }^{56} \mathrm{Fe}$ in shielding materials. As shown in Table 1[12], when the neutron energy is low, such as the neutron energy is $0.1 \mathrm{MeV}$, the total cross-section of the neutron interaction with ${ }^{56} \mathrm{Fe}$ is smaller than the total cross-section of its interaction with ${ }^{1} \mathrm{H}$ or ${ }^{12} \mathrm{C}$. The total cross-section is 3 orders of magnitude larger than the absorption cross-section. Only when the neutron energy is as low as less than $1 \mathrm{eV}$, the absorption cross-section can play a good shielding effect, so for the neutrons with energy of $0.1 \mathrm{MeV}$, the shielding performance of a single polyethylene material is always better than the same total thickness of iron-polyethylene combined shield. When the neutron energy is high, the total cross section of the interaction between neutron and ${ }^{56} \mathrm{Fe}$ is larger than the total cross section of its interaction with ${ }^{1} \mathrm{H}$ or ${ }^{12} \mathrm{C}$. For example, the total cross section of interaction between $14 \mathrm{MeV}$ neutron and ${ }^{56} \mathrm{Fe}$ is 2.6 barn, is larger than the total cross-section 0.69 barn or 1.30 bran that between $14 \mathrm{MeV}$ neutron and ${ }^{1} \mathrm{H}$ or ${ }^{12} \mathrm{C}$. When the neutron energy is above $2 \mathrm{MeV}$, the higher the energy of the neutron is, the smaller the total cross-section of the interaction with ${ }^{56} \mathrm{Fe}$ is, and the greater the thickness required for the iron to moderate the neutrons is. Therefore, in order to optimize the neutron shielding performance of the composite material shield, the optimal thickness ratio of iron to polyethylene increases with the energy of the neutron, this conclusion is consistent with the numerical simulation results. 
Table 1. Total cross section and absorb cross section for neutron interacting with different nuclides.

\begin{tabular}{|c|c|c|c|c|c|c|c|}
\hline \multirow{2}{*}{$\begin{array}{c}\text { Neutron } \\
\text { energy } / \mathrm{MeV}\end{array}$} & \multicolumn{3}{|c|}{ Absorption cross section / barn } & \multicolumn{3}{|c|}{ Total cross section / barn } \\
\cline { 2 - 7 } & ${ }^{1} \mathrm{H}$ & ${ }^{12} \mathrm{C}$ & ${ }^{56} \mathrm{Fe}$ & & ${ }^{1} \mathrm{H}$ & ${ }^{12} \mathrm{C}$ & ${ }^{56} \mathrm{Fe}$ \\
\hline $5 \times 10^{-3}$ & $7.37 \times 10^{-4}$ & $9.94 \times 10^{-6}$ & $9.58 \times 10^{-4}$ & & 19.8 & 4.72 & 5.78 \\
\hline 0.1 & $1.08 \times 10^{-4}$ & $9.00 \times 10^{-7}$ & $8.60 \times 10^{-5}$ & & 12.8 & 4.41 & 3.59 \\
\hline 2.1 & $3.44 \times 10^{-5}$ & $6.19 \times 10^{-6}$ & $1.73 \times 10^{-4}$ & & 2.83 & 1.89 & 3.77 \\
\hline 5 & $3.64 \times 10^{-5}$ & $2.88 \times 10^{-5}$ & $6.80 \times 10^{-4}$ & & 1.63 & 1.18 & 3.53 \\
\hline 14 & $2.98 \times 10^{-5}$ & $1.16 \times 10^{-4}$ & $7.30 \times 10^{-4}$ & & 0.69 & 1.30 & 2.60 \\
\hline
\end{tabular}

Figure 2(a) - (c) show that, when the total thickness of the combined shield is thin, for example, as the total thickness is $20 \mathrm{~cm}$, the optimum thickness ratio of iron to polyethylene exists only in theory, and the shielding performance of the combined shield has no obvious advantage over the shielding performance of iron alone, which is due to the moderation or absorption process of neutrons that require a shield to achieve a certain thickness. For relatively thin shields, the shielding effect depends mainly on the density of the shield material. Since the density of iron is much greater than that of polyethylene, so it should give priority to the use of high-density iron material when the total thickness of the shield material is limited and the shield quality is less limited.

\subsection{The effect of neutron energy on the optimal thickness ratio}

In order to obtain the optimal thickness ratio of iron and polyethylene in the combined shield with different energy neutrons, and to guide the radiation shielding practice, Figure 3 shows the relationship between the optimal thickness ratio with neutron energy when the thickness of the shield is $80 \mathrm{~cm}$.
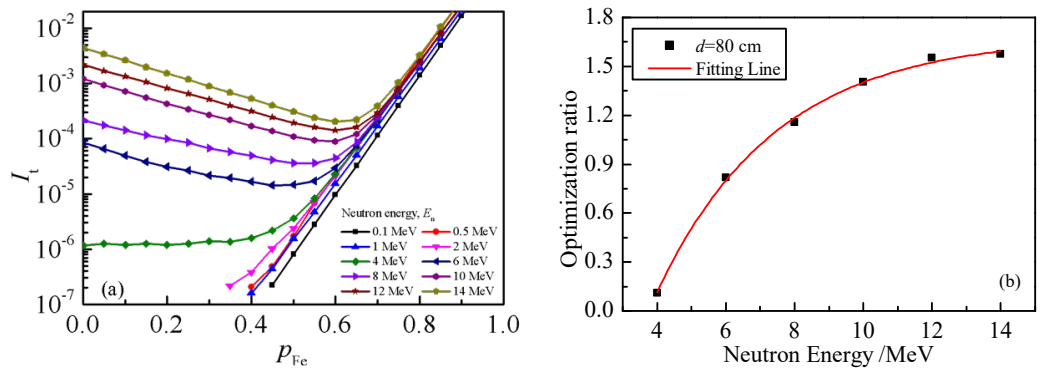

Fig. 3. Neutron shielding performance when thickness of combined shield structure $d=80 \mathrm{~cm}$. (a) Neutron transmission coefficient versus iron thickness proportion, (b) Optimal thickness ratio of iron to polyethylene as a function of neutron energy.

As shown in figure 3(a), the optimum thickness ratio of Fe to PE gradually decreases as the neutron energy decreases. When the neutron energy drops below $2 \mathrm{MeV}$, the better shielding effect can be obtained by using polyethylene alone. Figure 3(b) shows the relationship between the optimum thickness ratio of iron to polyethylene and neutron with energy range from $4 \mathrm{MeV} \sim 14 \mathrm{MeV}$. The results show that as the neutron energy gradually increases from $4 \mathrm{MeV}$ to $14 \mathrm{MeV}$, the optimum thickness ratio gradually approaches at 1.6: 1. The experssion is obtained by fitting the optimum thickness ratio with the neutron energy

$$
\delta_{\text {FePE }}=-4.94 \mathrm{e}^{-E_{\mathrm{n}} / 3.49}+1.68
$$

where, $\delta_{\mathrm{Fe} / \mathrm{PF}}$ represents the optimal thickness ratio of the iron to polyethylene layer in the combined shield, $E_{\mathrm{n}}$ is the average energy of the neutron to be shielded, in $\mathrm{MeV}$. As can be 
seen from figure 2, for the same energy of neutrons, when the thickness of the combined shield is respectively $40 \mathrm{~cm}, 60 \mathrm{~cm}$ and $80 \mathrm{~cm}$, the corresponding optimum thickness ratio is basically equal, so formula (3) is approximately suitable for the combined shield with total thickness of $40 \mathrm{~cm} \sim 80 \mathrm{~cm}$.

\section{Conclusions}

The combined shielding model was established, and the relationship between the optimal thickness ratio and the neutron energy was obtained by using the Monte Carlo simulation method. The results show that for the combined shield structure of iron and polyethylene, since iron mainly slows down and decelerates the energetic fast neutrons, polyethylene further slows down the moderating neutron and absorbs neutrons. Therefore, there will be an optimal thickness ratio in the combination of iron and polyethylene. When the neutron energy is high and the shielding structure is thick, with the increase of the ratio of the thickness of the iron layer to the total thickness of the combined shield, the neutron transmission coefficient of the combined shield firstly decreases and then increases gradually. When the thickness of the iron layer accounts for $30 \% \sim 60 \%$ of the total thickness, the shielding effect is relatively good; the optimal thickness ratio of iron to polyethylene is related to the neutron energy and the total thickness of the combined shield; When the combined shielding total thickness is in the range of $40 \mathrm{~cm} \sim 80 \mathrm{~cm}$, as the neutron energy increases from $4 \mathrm{MeV}$ to $14 \mathrm{MeV}$, the optimal thickness ratio gradually increases from 0.1 and approaches 1.6: 1. The optimal thickness ratio of iron and polyethylene simulated in this paper can provide theoretical reference for design of combined shield of iron and polyethylene in radiation protection.

\section{References}

1. Y.H. Zuo, H.G. Xie, J.H. Zhu, Modern Applied Physics, 8, 020702 (2017)

2. L. Huo, J.L. Liu, Y.H. Ma, Radiation Dose and Protection (Publishing house of electronics industry, Beijing, 2015)

3. V.P. Singh, N. M. Badiger, Annals of Nuclear Energy, 64, 301 (2014)

4. H. Wu, F. Zhang, Y.F. Li, Z.Y. Han, M.X. Gu, Radiation Protection, 37, 94 (2017)

5. X.M. Zeng, P. Zhou, P.Z. Qin, M. Bao, G.S. Guo, Z.Y. Xu, Nuclear Techniques, 34, $188(2011)$

6. W.F. Yang, X.Y. Wu, Y. Liu, Nuclear Power Engineering, 36, 58 (2015)

7. Z.W. Han, W.L. Luan, Y.L. Han, Y. Zhang, G.Z. Wu, Nuclear Techniques, 38 (2015)

8. Z.Q. Shen, Q. Kang, J. Xu, Z.G. Wang, N. Lv, Progress Report on China Nuclear Science \& Technology, 3, (2013)

9. H.Z. Yang, Z.E. Yao, Journal of Lanzhou University( Natural Sciences), 33, 60 (1997)

10. P. Luo, Z.E. Yao, Y. Lian, J.F. Hu, S.J. Jun, Nuclear Techniques, 31, 756 (2008)

11. J.F. Briesmeister MCNP-A general Monte Carlo N-Particle transport code, Ver.4B, LA-12625-M (Los Alamos Scientific Laboratory, New Mexico, 1997)

12. D.F. Tian, W.L. Sun, Excitation functions in neutron-induced reactions (National Defense Industry Press, Beijing, 2006) 